\section{S116 MICRORNA REGULATION OF TOLL-LIKE RECEPTOR 7 FUNCTION IN SEVERE ASTHIMA: RELEVANCE TO VIRAL RESPONSES}

doi:10.1136/thoraxjnl-2012-202678.121

H Rupani, P Dennison, N Jayasekera, T Sanchez-Elsner, P H Howarth. Clinical and Experimental Sciences, Faculty of Medicine, University of Southampton, Southampton, United Kingdom

Background and Aims Viral-induced disease exacerbation is common in asthma and studies have identified that both bronchial epithelial cells and alveolar macrophages (AM) from asthmatics have a reduced interferon (IFN) response to rhinovirus infection. The mechanism behind this defect is unclear. As asthmatic peripheral blood mononuclear cells have been reported to have defective tolllike receptor (TLR) 7 function, we investigated the expression of microRNAs (miRNAs) in AM from healthy control (HC) and severe asthma (SA) volunteers with relevance to TLR7-viral interactions. MicroRNAs are non-coding RNAs that down-regulate gene expression by suppressing translation. We identified and focused on 3 miRNAs that could target TLR7. Additionally, we investigated if manipulating the expression of these miRNAs can ameliorate the defective IFN response in AM.

Methods $26 \mathrm{HC}$ and 30 patients with SA (BTS Step 4/5) were recruited for bronchoscopy. AM were isolated from bronchoalveolar lavage using the adherence to plastic technique. Expression of miRNAs and TLR7 was determined by qRT-PCR and western blotting. AM were transfected with a combination of antagomirs, specifically directed against the 3 miRNAs, and then treated with imiquimod (5ug/ml), a TLR7 agonist, or human rhinovirus-16 (HRV16) and IFN- $\beta$ expression was determined after 24 hours using qRT-PCR and ELISA.

Results Expression of all three miRNAs was significantly increased in SA compared to HC TLR7 mRNA was found to be significantly reduced in AM from volunteers with SA compared to HC. Western blotting confirmed reduced expression of TLR7 protein in AM from SA compared to HC. Compared to mock transfected AM, AM transfected with the 3 antagomirs showed significantly increased imiquimod-induced IFN- $\beta$ mRNA and protein expression and significantly increased HRV16-induced IFN- $\beta$ mRNA production.

Conclusion TLR7 expression is significantly reduced in SA compared to HC. The differential expression of the miRNAs identified may lead to impaired viral sensing by asthmatic AM and contribute to the defective IFN response to rhinovirus. Importantly, TLR7 induced IFN- $\beta$ production by human AM can be significantly augmented by inhibition of these miRNAs. The identification of these miRNAs and our ability to manipulate their expression in human AM offers the potential for future miRNA-based therapies in asthma.

\section{S117 EFFECTS OF HYPOXIA ON EOSINOPHIL APOPTOSIS, EFFEROCYTOSIS AND SENSITIVITY TO GLUCOCORTICOSTEROIDS}

doi:10.1136/thoraxjnl-2012-202678.122

L Porter, AS Cowburn, N Farahi, CA Fiddler, AM Condliffe, ER Chilvers. University of Cambridge, Cambridge, England

Many tissues including the skin, intestinal epithelium and potentially the airway operate at 'physiological' levels of tissue hypoxia with normal $\mathrm{PO}_{2}$ values below $3 \mathrm{KPa}$. Both sterile and non-sterile inflammation exacerbates the degree of tissue hypoxia and predicates the need for granulocytes including eosinophils to operate efficiently under hypoxia. In these experiments we have examined the effects of hypoxia on eosinophil longevity and show that a $\mathrm{PO}_{2}$ below $3 \mathrm{KPa}$ severely attenuates the pro-apoptotic effect of dexamethasone.
Human blood eosinophils were prepared from healthy donors using hetastarch-sedimentation and EasySep ${ }^{\circledR}$-immunomagenetic beads, and cultured in RPMI $+10 \%$ autologous serum for $6-24 \mathrm{~h}$. Apoptosis and efferocytosis were quantified using standard morphology, AnV/PI staining and myeloperoxidase counter-stain methods. Hypoxic incubation $(\mathrm{H})$ (media $\mathrm{PO}_{2} 2.9 \pm 0.1 \mathrm{KPa}$ ) caused a marked survival response in eosinophils compared to normoxia $(\mathrm{N})$ (\% apoptosis at $24 \mathrm{~h}: \mathrm{N} 16.3 \pm 3.0 \%$; $1.2 \pm 0.2 \%, \mathrm{n}=5$ ), which was of similar magnitude to that observed with IL-5 (N $4.2 \pm 0.3 \%$ ). This hypoxic survival effect was mimicked by the iron chelator/2-oxyglutarate analogue DFO (10 mM) and DMOG (1 mM) and blocked in a concentration-dependent manner by the protein synthesis inhibitor cycloheximide. In preliminary experiments hypoxia also independently reduced the capacity of monocyte-derived macrophages to phagocytose apoptotic eosinophils as seen previously with neutrophils. Most strikingly, hypoxic incubation also reduced the normal pro-apoptotic effect of dexamethasone $(1 \mu \mathrm{M})(\%$ apoptosis at $24 \mathrm{~h}$ : $\mathrm{N}+\operatorname{Dex} 27.6 \pm 3.9 \%, \mathrm{H}+\operatorname{Dex} 11.2 \pm 2.9 \%)$. qPCR analysis of the glucocorticosteroid-dependent gene GILZ and the hypoxia-HIF-1 $\alpha$-dependent gene GLUT1 demonstrated that the above suppression of dex-induced eosinophil apoptosis was not a consequence of the inhibition of steroid-induced transcriptional

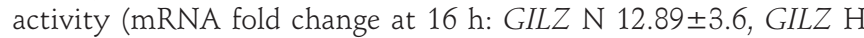

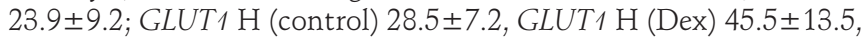
$\mathrm{n}=3$ ).

These data suggest that hypoxia can cause a profound effect on eosinophil longevity and macrophage uptake, and render these cells partially resistant to the pro-apoptotic effects of dexamethasone. This may impede the effective resolution of eosinophilic inflammation in vivo.

\section{S118 IL-13 INDUCED MOUSE AIRWAY INFLAMMATION INDUCES AN INCREASE OF SOLUBLE ADAMI3 IN BRONCHOALVEOLAR LAVAGE FLUID, WHICH IS ENZYMATICALLY ACTIVE AND ASSOCIATED WITH BRONCHIAL HYPERRESPONSIVENESS}

doi:10.1136/thoraxjn-2012-202678.123

1.2ER Davies, ${ }^{2} \mathrm{G}$ Chen, ' $\mathrm{ST}$ Holgate, 'DE Davies, ${ }^{2} \mathrm{JA}$ Whitsett, ${ }^{1,2} \mathrm{HM}$ Haitchi. ${ }^{1}$ Clinical and Experimental Sciences, Faculty of Medicine, University of Southampton, Southampton, UK; ${ }^{2}$ Division of Pulmonary Biology, Cincinnati children's Hospital Medical Center and University of Cincinnati College of Medicine, Cincinnati, USA

Rationale The asthma susceptibility gene ADAM33/Adam33 is associated with bronchial hyperresponsiveness(BHR) in humans and mice. Soluble ADAM33 is increased in bronchoalveolar lavagefluid (BALF) of allergic asthma patients (Lee JY et al, AJRCCM 2006 Apr1; 173(7):729-35). Its levels correlate with declining FEV \%, suggesting a role in airway remodelling in asthma. Maternal allergy orexogenous IL-13 suppresses Adam33/ADAM33mRNA expression but enhances ADAM33 protein processing in human embryonic and juvenile mouse lungs (Haitchi HM et al, JACI. 2009 Sep; 124(3):590-7, 597). We hypothesise thatconditional expression of IL-13 in mouse lungs induces the enzymatically active, soluble form of ADAM33 in BALF, which is associated with BHR.

Methods IL-13 expression wasinduced using Doxycycline in CCSP-rtTA/Otet-Il-13 double-transgenic (dTg) mice. Methacholine challenge and lung function measurements were performed and lungswere harvested for mRNA extraction and immunohistochemistry (IHC). BALF was obtained forWestern-blotting for ADAM33 and testing of ADAM33 enzymatic activity using a fluorescenceresonance energy transfer (FRET) peptide assay.

Results There was asignificant increase in BHR to Methacholine in IL-13 expressing double transgenicmice. IHC showed an increase inbronchial smooth muscle in lungs of double transgenic mice. Similar to the RTqPCR findings in humanembryonic and juvenile mouse 